\title{
Top ten universities from CWTS Leiden Ranking 2018
}

The CWTS Leiden Ranking 2018 (http://www.leidenranking.com/) has just been released. The meticulously curated bibliometric data made available by the Centre for Science and Technology Studies (CWTS), Leiden, the Netherlands, allows comparative evaluation of the scientific performance of nearly 1000 major universities worldwide.

Here we follow the same methodology that was reported earlier in these pages ${ }^{1}$. The 2018 ranking is based exclusively on the Web of Science (WoS) database of Clarivate Analytics, Philadelphia, PA, USA, and covers 938 universities from 55 different countries. All universities worldwide with more than 1000 fractionally counted WoS indexed core publications during the period 2013-16 are included in the ranking.

In a typical assessment window (say, 2013-2016), an institution will have published a total number of papers or articles, $P$ (fractionally counted), and received a total number of citations, $C$. $P$ can be taken as the indicator or proxy measure for the size of the unit and $C$ is the total impact of its published research respectively. Over the years, CWTS has found that the proportion of the top $10 \%$ publications, PP (top 10\%) is arguably the most robust, size-independent proxy or indicator for the quality of publications. This is the proportion of the publications of a university that, compared with other similar publications, belongs

Table 1. The top ten universities derived from the second-order indicator $X=q^{2} P$

\begin{tabular}{rlrrrr}
\hline Rank & University & Year & $P$ & $q$ & $X$ \\
\hline 1 & Harvard University, USA & $2012-15$ & 32,912 & 2.24 & $164,454.68$ \\
2 & Stanford University, USA & $2013-16$ & 15,364 & 2.24 & $77,068.71$ \\
3 & Massachusetts Institute of & $2011-14$ & 10,226 & 2.54 & $66,194.01$ \\
& $\quad$ Technology, USA & & & \\
4 & University of California, Berkeley, USA & $2012-15$ & 12,156 & 2.18 & $57,540.27$ \\
5 & University of Oxford, UK & $2013-16$ & 14,505 & 1.88 & $51,460.10$ \\
6 & University College London, UK & $2013-16$ & 14,203 & 1.78 & $45,121.33$ \\
7 & University of California, & $2010-13$ & 14,012 & 1.78 & $44,536.31$ \\
& $\quad$ Los Angeles, USA & & & & \\
8 & University of Michigan, USA & $2012-15$ & 18,121 & 1.57 & $44,463.68$ \\
9 & University of Cambridge, UK & $2013-16$ & 13,103 & 1.84 & $44,205.28$ \\
10 & Johns Hopkins University, USA & $2013-16$ & 16,831 & 1.60 & $43,243.63$ \\
\hline
\end{tabular}

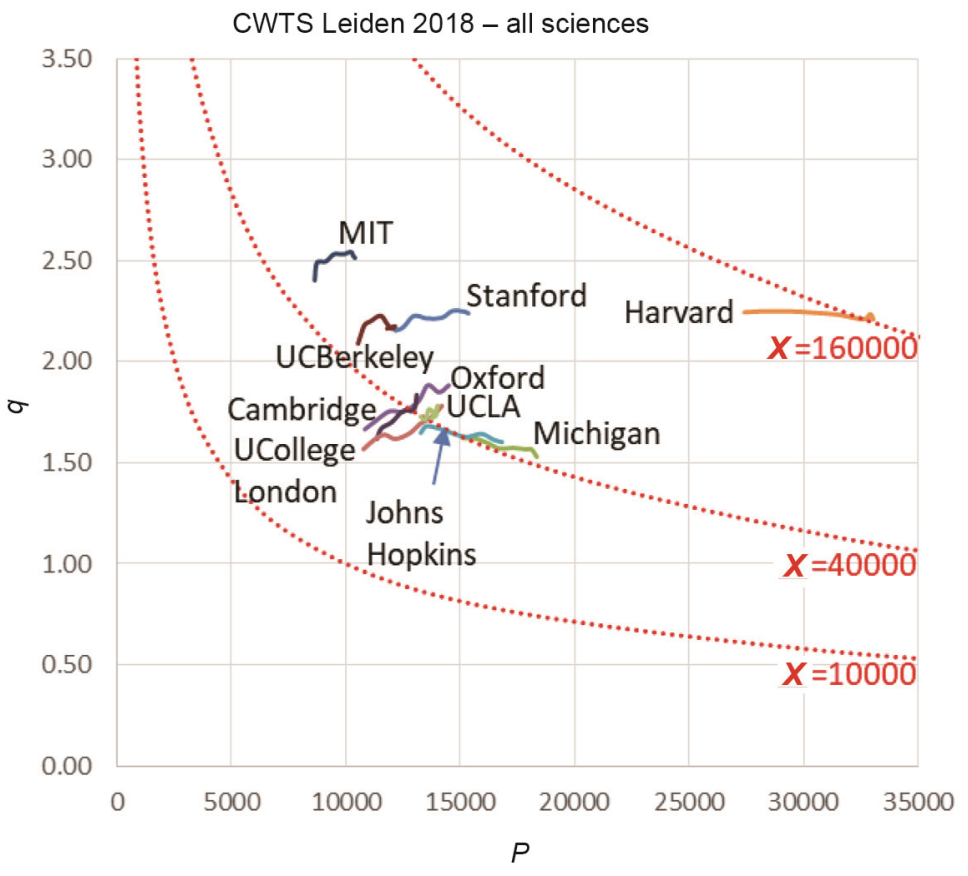

Figure 1. Performance trajectories of the top 10 universities from the 2006-2009 to the 2013-2016 window. 


\section{CORRESPONDENCE}

to the top $10 \%$ most frequently cited. It therefore has a normalizing effect across fields, publication year and document type. The ratio $q=P P$ (top $10 \%$ ) $/ 10$, allows one to fractionalize this proxy, such that a value of 1.00 is the expected global norm.

If we consider $q$ to be the quality indicator, and $P$ to be the zeroth-order indicator of performance, then it is possible to combine this to obtain a first-order indicator of performance $q P$ and a secondorder indicator of performance $X=q^{2} P$ (ref. 2). In this manner, the quantity term $(P)$ and the quality term $(q)$ can be integrated into a single composite term that serves as the best size-dependent proxy for total performance in research context.

Table 1 lists the top ten universities derived from the second-order indicator $X=q^{2} P$ for all sciences taken together.
They are entirely from 'both sides of the pond'. Harvard is twice as big as the next in line and four times bigger than the others in the list. Figure 1 shows the performance trajectories of these universities in a quality-quantity space as we move from the 2006-2009 to the 20132016 window. Since the global norm for $q$ is 1 , all the universities perform above global norm. Harvard's trajectory is flat. The three entries from the United Kingdom, i.e. University of Oxford, University College London and University of Cambridge show the most promising trajectories, where rapid growth in size of output does not compromise quality as measured by the $q$ indicator.

A unique feature of the Leiden ranking is that it allows anyone to build a multidimensional perspective on university performance from the size-dependent and size-independent indicators which are available for download. This feature is exploited here to visualize how the top 10 world universities can be identified depending on the choice of primary indicators.

1. Prathap, G., Curr. Sci., 2014, 106, 14671468.

2. Prathap, G., Scientometrics, 2011, 87(3), 515-524.

GANGAN PRATHAP

Vidya Academy of Science and

Technology,

Thrissur 680 501, India and

A. P. J. Abdul Kalam Technological

University,

Thiruvananthapuram 695 016, India

e-mail:gangan@vidyaacademy.ac.in

\section{Agrobio-cultural diversity of alder based shifting cultivation practiced by Angami tribes in Khonoma village, Kohima, Nagaland}

North East India is one of the culturally diverse regions in the world inhabited by more than 200 tribes in eight states. Also, the region is one of the biodiversity hot spots of the world. The region is endowed with rich floral, faunal and sociocultural diversity. These tribes have originated from the ethnic groups of Tibeto-Burmese and Indo-Mongoloids ${ }^{1}$. The tribal communities of this region live in hilly areas and depend on forest resources for their livelihood. Shifting cultivation is the major agricultural land use system in undulating hilly terrains of this region. Shifting cultivation (jhum cultivation) by these communities is practised in a cyclic manner of slash and burn, crop cultivation followed by abandoning of the land and subsequent shift from one place to another. The previously abandoned land is revisited for crop cultivation after 15-20 years of jhum cycle or 3-5 years in the current scenario of short jhum cycles. Shifting cultivation practice in North East (NE) India is profoundly linked to the socio-economic and cultural customs of these tribes. The agro-biodiversity of jhum cultivation is as high as the cultural diversity of the region $^{2}$. Different tribes celebrate cultural festivals before growing and after har- vesting the crops in their own customary manner. This tradition in NE India has conserved rich cultural diversity since time immemorial. However, continuous slash and burn of forest land has resulted in significant loss of rich natural biodiversity, soil erosion and land degradation $^{3}$.

Khonoma, the first Green Village of Asia, is located about $20 \mathrm{~km}$ from the state capital of Nagaland and the name is derived from Khwunoria (the Angami

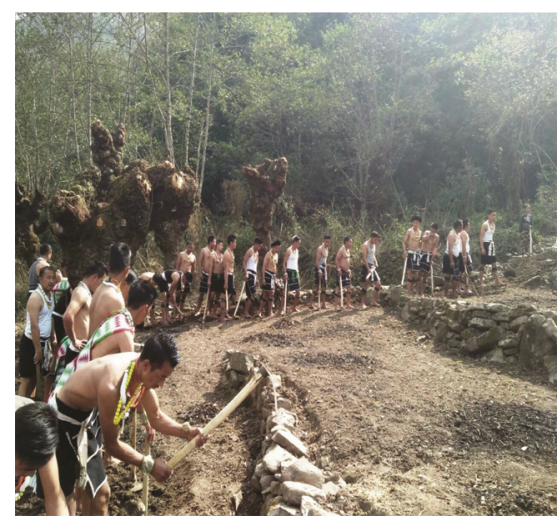

Figure 1. Preparation of land for crop cultivation (Courtesy: Mr Kedocakho, Khonoma Village). name of Gaultheria fragrantissima, the Indian wintergreen). The village is estimated to be around 700 years old and spread over an area of $123 \mathrm{~km}^{2}$. The total population of the village is about 3000 , settled in 600 households ${ }^{4}$. Khonoma village is well known for its forests and a unique form of agriculture, including oldest wet terrace cultivation in the region. The village is well known for Alnus nepalensis (Himalayan alder)based shifting cultivation which is an

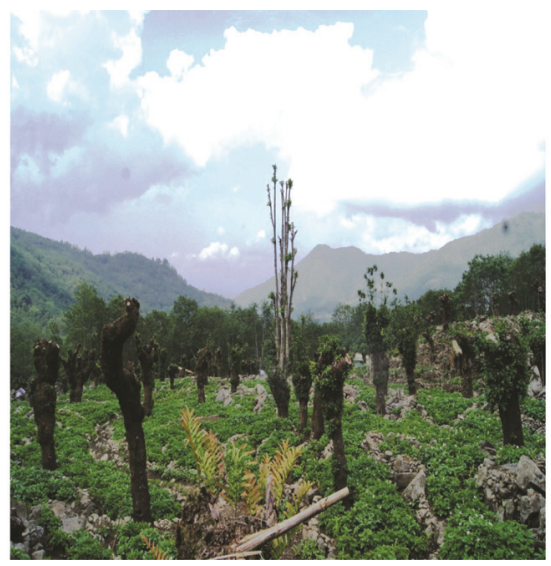

Figure 2. Potato cultivation in alder grown jhum fields (Photograph: Dr Krishna Giri). 